\title{
Processing strategies in sentence comprehension
}

\author{
JILL T. WANNEMACHER \\ Bryn Mawr College, Bryn Mawr, Pennsylvania 19010
}

\begin{abstract}
Response latencies in sentence-picture verification tasks were compared as a function of whether a mismatch was located in the logical subject (LS), verb (V), or logical object (LO) of the sentence. Sentences were presented auditorily and varied in voice and reversibility. The comparison process for nonreversibles was clearly serial self-terminating: latencies for both actives and passives were ordered $\mathrm{LS}<\mathrm{V}<\mathrm{LO}$, or, after practice with a small number of mismatch types, $\mathrm{LS}<\mathrm{LO}<\mathrm{V}$. Latencies for reversibles were ordered $V<L S=L O$, suggesting either a verb-first comparison process or an LS-V.LO comparison process which did not terminate with a subject-mismatch because of the confusability of the subject and object. The results attest to the importance of considering the "naturalness" of stimuli in sentence processing tasks, and the flexibility of subjects' encoding and comparison strategies both within and across task contexts.
\end{abstract}

Two recent experiments have attempted to delineate the encoding and comparison strategies used in sentence-picture verification tasks. Wannemacher (1974) and Glucksberg, Trabasso, and Wald (1973) both varied the location of the mismatch between a sentence and picture, and compared the latencies of "same-different" responses as a function of whether the mismatch occurred in the sentence subject, verb, or object. By examining the relative latencies to the various mismatch types, it was thus possible to determine the type of comparison strategy used (e.g., serial or parallel, exhaustive or self-terminating), as well as whether sentence constituents were processed in their surface structure order, or recoded into a deep structure format.

Wannemacher presented sentences auditorily, and pictures immediately preceded (Experiment I) or were simultaneous with (Experiment II) the sentences. In both tasks, subjects adopted a serial self-terminating comparison strategy and a surface structure encoding strategy: comparison terminated as soon as a mismatch was encountered, and actives were processed in the order logical subject-verb-logical object (LS-V-LO), whereas the order for passives was reversed (LO-V.LS). Since the comparison process was initiated before the entire sentence had been presented, the serial stage analysis proposed by Wannemacher represented the sequence as a processing loop consisting of an encoding, a comparison, and a response-decision stage, applied to each lexical constituent individually.

Glucksberg et al. obtained very different results, but their procedure differed from Wannemacher's in several ways: (1) sentences were presented visually and either preceded or followed the pictures; (2) subjects were given unlimited time for encoding the first stimulus input; (3) false sentences were of three types, containing

This research was supported by grants from the National Institute of Child Health and Human Development: Experiment I was part of a doctoral dissertation submitted to Brown University, supported by PHS Grant HD 05531 to Peter D. Eimas. Experiment II was supported by PHS Grant HD 08628 to the present author. a single mismatch in either the subject (s), verb (v), or object (o), whereas Wannemacher presented either one $(\mathrm{s}, \mathrm{v}$, or 0$)$, two (s-v, v-o, s-o) or three (s-v-o) mismatches; (4) the set of nouns and verbs from which descriptions and pictures were drawn was much smaller than Wannemacher's; (5) Glucksberg et al.'s subjects were more highly practiced.

Glucksberg et al.'s results supported the hypothesis that the comparison operation in verification tasks is serial self-terminating, but the sentence constituents were not processed in their surface structure order: false latencies from fastest to slowest were ordered (1) verb, (2) GS (grammatical subject or first noun), (3) GO (grammatical object or second noun). Glucksberg et al. therefore proposed a processing model which differs from Wannemacher's: (1) subjects are assumed to encode sentences in a list format ordered V-GS-GO; (2) in the picture-sentence condition it is assumed that sentences are fully encoded; (3) voice and reversibility information are assumed to be unnecessary unless all three constituents match, therefore to affect only true responses.

Both Wannemacher and Glucksberg et al. suggested that subjects may vary their encoding and comparison strategies as the demands in verification tasks are changed. In Wannemacher's studies, the auditory presentation of sentences, combined with limited encoding time, undoubte dly maximized the efficiency of a surface structure encoding strategy, whereas Glucksberg et al.'s procedure may have maximized the efficiency of a strategy which focused on the verb: (a) the verb was the least variable constituent since all sentences and pictures were constructed from a stimulus set of three verbs, four agents, and seven objects; (b) subjects had ample time to encode the first stimulus in a verb-first list format.

Assuming that human subjects are, in fact, flexible in their processing strategies, the usefulness of general comprehension models depends on being able to identify the task variables that elicit particular processing 
Table 1

Mean RT, Standard Error of the Mean (in Milliseconds), and Percentage Errors for Each Treatment Type

\begin{tabular}{clrcc}
\hline & & $\begin{array}{c}\text { Mean } \\
\text { RT }\end{array}$ & $\begin{array}{c}\text { SE } \\
\text { Mean }\end{array}$ & $\begin{array}{c}\text { Percent } \\
\text { Error }\end{array}$ \\
\hline True & & 1107 & 86 & 6.7 \\
False & s-v-o & 902 & 67 & 1.4 \\
& s-v & 937 & 40 & 0 \\
& $\mathrm{~s}-\mathrm{o}$ & 1017 & 48 & 2.8 \\
Reversible & $\mathrm{s}$ & 1063 & 28 & 11.4 \\
& $\mathrm{v}-\mathrm{o}$ & 964 & 52 & 1.4 \\
& $\mathrm{v}$ & 958 & 60 & 4.2 \\
& $\mathrm{o}$ & 1082 & 67 & 12.8 \\
True & & 928 & 80 & 3.6 \\
False & $\mathrm{s}-\mathrm{v}-\mathrm{o}$ & 841 & 51 & 0 \\
& $\mathrm{~s}-\mathrm{v}$ & 931 & 64 & 2.8 \\
& $\mathrm{~s}-\mathrm{0}$ & 868 & 58 & 2.8 \\
Nonreversible & $\mathrm{s}$ & 851 & 75 & 1.4 \\
& $\mathrm{v}-\mathrm{o}$ & 983 & 66 & 0 \\
& $\mathrm{v}$ & 978 & 58 & 7.1 \\
& $\mathrm{o}$ & 1027 & 84 & 8.5 \\
\hline
\end{tabular}

strategies, and thus to evaluate the generality of the various strategies. The experiments which follow were designed to determine the nature of subjects' encoding and comparison strategies when pictures follow sentences in a task context more similar to a natural language processing situation than that of Glucksberg et al.: when sentences are auditory and must be encoded quickly, and when the lexical constituents are drawn from very large stimulus sets. In addition, the influence of the number of false sentence types was assessed by presenting either seven (Experiment I) or only three (Experiment II) mismatch types. Of particular concern are (1) whether in such contexts subjects inevitably adopt a verb-first processing strategy, (2) whether experience with a small set of sentence types leads to strategy changes, and (3) whether a semantic variable like reversibility influences only true response latencies, as Glucksberg et al. suggest, or false response latencies as well.

\section{EXPERIMENT 1}

\section{Method}

For a detailed description of the stimuli, apparatus, and design used in Experiment I, the reader should refer to Wannemacher (1974). Three independent variables were manipulated within subjects: voice, reversibility, and treatments (true sentences and seven mismatch types). Each subject heard a total of 280 sentences, representing 70 of each of four sentence types: active reversible (AR), e.g., "the boy is chasing the dog"; active nonreversible (ANR), e.g., "the girl is picking the flowers"; passive reversible (PR), e.g., "the dog is being chased by the boy"; and passive nonreversible (PNR), e.g., "the flowers are being picked by the girl". Within each group of 70 half of the sentences matched the following pictures and half mismatched, and there were an equal number (5) of each of seven mismatch types: $s, v, o, s-v, v-0, s-v, s-v-o$. The pictures were $35-\mathrm{mm}$ slides of colored cartoon-like drawings, projected on a screen in front of the subject.

On each trial, subjects listened to a tape-recorded sentence, whose offset initiated presentation of a picture. An RT clock was activated by onset of the picture, and terminated by the subject's pushbutton response ("same" or "different"). The pairing of response hand to "same" and "different" was varied by instructing alternate subjects to respond "same" with the preferred hand. The subjects were seven introductory psychology students who were paid for participating in two 60-min sessions.

\section{Results and Discussion}

Mean RTs were subjected to a four-way analysis of variance (subjects by voice by reversibility by treatments), with the appropriate Subjects by Effects interactions serving as denominators for each $F$ ratio. Significant main effects were obtained for reversibility $[F(1 / 6)=37.89, \quad p<.001]$ and treatments $[F(7 / 42)=3.85, p<.005]$. The only significant interaction was Reversibility by Treatments $[F(7 / 42)=5.27, p<.005]$. Table 1 shows the mean RT, standard error of the mean, and percentage of errors for each treatment type as a function of reversibility. Data for actives and passives were combined since voice was not significant either as a main effect or in any interaction. The labeling of the mismatch types refers to the deep structure (logical) function of the lexical constituent. The overall error rate was $4.6 \%$, with a range from $3.2 \%$ to $7.1 \%$. Only correct responses were included in the RT analyses.

The Comparison Strategy. Considering only false response latencies, the pattern of RTs to mismatches in nonreversibles in Table 1 indicates that subjects adopted a serial self-terminating comparison strategy in the order logical subject-verb-logical object; RT was fastest to mismatches involving the subject (s-v-o, s-v, s-o, s) slower when the first (or only) mismatch occurred in the verb ( $v-0, v)$, and slowest when only the object mismatched (o). Duncan's multiple range tests revealed that all mismatches involving the subject were processed significantly faster than those involving only the object, and, with the exception of s-v mismatches, significantly faster than v- and v-o-mismatches. The data for nonreversibles thus clearly indicate that a verb-first comparison strategy is not inevitably adopted when pictures follow sentences.

The relationship between true and false response latencies in nonreversibles is similar to that obtained by Glucksberg et al.: several of the mismatch types were processed more quickly, and several more slowly, than true sentences. In Table 1 the significant differences were $\mathrm{T}<0, \mathrm{~T}>\mathrm{s}-\mathrm{v}-\mathrm{o}, \mathrm{p}<.05$. This finding can be reconciled with a self-terminating model when the total processing sequence is considered, and will be more fully discussed below.

The pattern of latencies to reversibles in Table 1 does suggest a comparison strategy that focused on the verb: RTs to all mismatches involving the verb (s-v-o, s-v, v-o, v) were statistically equal, and significantly faster than RTs to s- or o-mismatches $(p<.05)$, which were equal. 
The data do not, however, support Glucksberg et al.'s finding of a V-GS-GO order of comparison and, in fact, are compatible with several comparison strategies. The most obvious interpretation is that subjects check the verb first, terminating the comparison if a mismatch is found. If the verb matches, however, it is not clear whether (a) both the $s$ and o are then compared, because of the possibility of confusing them, or (b) whether after checking the verb either $s$ or $o$ could be compared second, with comparison terminating on a mismatch. Either strategy would lead to equal RTs to s- and o-mismatches, but only the latter would also lead to faster RTs to s-o-mismatches (where both $\mathrm{s}$ and o differ) than to single mismatches in either the $s$ or $o$. This difference does occur in Table 1 , but failed to reach statistical significance.

Either verb-first comparison strategy can explain the latency pattern for reversibles, but both require the assumption that the order of comparison for reversibles differed from that for nonreversibles. While this is possible, an alternative interpretation might be that reversibility influenced the criterion for terminating the comparison, rather than the order of comparison. Subjects might process both reversibles and nonreversibles in an LS.V.LO order, but in reversibles not terminate with a subject-mismatch because of the confusability of $s$ and $o$, but go on to check the verb as well. If the verb mismatches comparison terminates, but if it matches the object is also compared. Note, however, that this does not necessarily entail determining the grammatical relations. There is sufficient information for a false response without that since no false sentence actually reversed the relations pictured. Both s- and o-mismatches would thus require three comparisons, while verb-mismatches require only two, resulting in faster RTs. Such a processing strategy would produce the latency differences in Table 1, but without requiring subjects to use a different order of comparison for reversibles and nonreversibies.

The Encoding Format. The constituents of passive nonreversibles (PNRs) were clearly not compared in a passive surface structure order, but neither were PNRs recoded into an active format prior to the comparison operation: (a) RTs to PNRs, whether true or false, were generally no slower than those to ANRs, a fact not revealed in Table 1 since the data for actives and passives were combined, and (b) subjects did not have time to recode passives prior to the onset of the picture. The results thus suggest the syntactic factor of voice was bypassed in processing NRs, and sentence-picture pairs were compared on the basis of semantic information, or a semantic "mapping," alone (cf. Bever, 1970; Huttenlocher \& Higgens, 1972).

In the case of reversibles, the encoding format is not clear, as was noted above. False responses were no longer to passive than to active reversibles, which suggests voice did not influence the encoding or comparison process.
However, true responses were longer for passives than for actives (true PR = $1149 \mathrm{msec}$; true AR $=1064 \mathrm{msec}$ ), which suggests that voice information could be bypassed unless ali three constituents were found to match, in which case the response-decision took longer for passives than for actives. One possible explanation for this is that reversible sentences, like nonreversibles, are encoded as heard in a surface structure format (i.e., LS-V-LO for actives, LO-V-LS for passives). Pictures, however, are always encoded in the same format, regardless of the voice of the preceding sentence. If the comparison reveals a mismatch subjects can respond false without considering the voice of the sentence, since one of the constituents is clearly different; thus voice does not affect false response latencies. If the comparison locates the three constituents presented in the sentence, however, a response-decision requires matching up subjects and objects, which requires more time when the preceding sentence constituents were ordered LO-V-LS.

Factor-Stage Relations in Processing. The latency data for NRs in Experiment I suggest that, when pictures follow sentences, the encoding, comparison, and response-decision processes may overlap. That is, because the picture constituents are presented in parallel, encoding and comparison of the second constituent can occur before a response-decision about the first has been reached. Evidence for this comes from the relative latencies to true and false nonreversibles: the comparison was clearly self-terminating, but RTs to mismatch types requiring only two comparisons were generally no faster than those to true sentences, which required three comparisons. To reconcile this finding with a self-terminating model, one must assume that (a) stages in the processing sequence overlap, and (b) subjects are initially "set" to make a true response, and false responses require resetting a "truth index" (cf. Clark \& Chase, 1972; Olson \& Filby, 1972; Trabasso, 1972). A false response is thus faster than a true response only when a mismatch decision can be initiated early in the comparison process, presumably because changing the truth index to false requires more time than checking one more constituent and responding true. In reversibles, of course, true response-decisions take longer, since a match of all the constituents requires the further consideration of sentence voice, which is not true for nonreversibles.

The results thus indicate subjects do not inevitably adopt a verb-first processing strategy when latency to a visual input is measured, and that reversibility does influence the processing of false, as well as true, sentences. A question still remaining, however, is which of the task variables in the Glucksberg et al. study led to a verb-first processing strategy in both reversibles and nonreversibles. The results of Experiment I rule out the visual presentation of the second stimulus input. Experiment II was conducted to determine whether reducing the number of mismatch types from seven to 
three $(s, v$, or $o)$ would induce a verb-first processing strategy. The procedure thus replicated that of Glucksberg et al. in the number and types of mismatches, but differed in that (a) sentences were heard rather than seen, and (b) sentence and picture constituents were drawn from a larger stimulus set.

\section{EXPERIMENT II}

\section{Method}

Each subject heard a total of 120 sentences, representing 30 of each of the four sentence types (AR, PR, ANR, PNR). Half of the sentences matched the following picture and half mismatched, and there were an equal number (5) of each of the three mismatch types: $s, v$, or $o$. The sentences were grouped into five blocks of 24 , each containing an equal number of every sentence and mismatch type. Each picture was seen twice, paired once with a true and once with a false sentence. In all other respects, the procedure was identical to that in Experiment 1. The subjects were 14 introductory psychology students who were paid for participating in a single 60 -min session.

\section{Results and Discussion}

The major findings in Experiment II are easily summarized: reducing the number of mismatch types did not induce the adoption of a verb-first processing strategy for nonreversibles; it did, however, result in a clear practice effect, with the pattern of response latencies changing markedly over trials. To facilitate analysis, the data were therefore considered in two parts: early (Blocks 1 and 2) and late (Blocks 3-5), and separate analyses of variance were performed on each part. Table 2 shows the mean RT, standard error of the mean, and percentage of errors for each treatment type as a function of reversibility, voice, and practice (early vs. late). The overall error rate was $4.2 \%$, with a range from $.8 \%$ to $9.2 \%$.
Processing Strategies on Early Trials. The analysis of the early latencies (Table $2 \mathrm{~A}$ ) revealed significant effects of reversibility $[F(1 / 13)=49.95, p<.001]$, treatments $[F(3 / 39)=7.02, \quad p<.001]$, and Reversibility by Treatments $[F(3 / 39)=7.60, p<.001]$. The nature of these effects can be seen in Figure 1A, where those data points not reliably different have been pooled. In NRs subjects clearly adopted a serial self-terminating strategy in the order LS-V-LO, regardless of sentence voice. Latencies to the three mismatch types were significantly different $(p<.05)$, and the relationship between true and false latencies replicates that in Experiment I $(s<\mathrm{T}<0)$. In reversibles true responses were significantly faster than those to all mismatch types, suggesting an exhaustive comparison of the constituents prior to a response decision. In view of the variability of the latencies for the three mismatch types, however, it would be imprudent to speculate more specifically about the nature of the comparison process.

Processing Strategies on Later Trials. The analysis of the late latencies (Table 2B) showed a significant effect of reversibility $[F(1 / 13)=35.53, p<.001]$ and significant interactions between treatments and reversibility $[F(3 / 39)=18.59 . p<.001]$, voice and reversibility $[F(1 / 13)=7.73, p<.05]$, and voice and treatments $[F(3 / 39)=4.11, p<.05]$. A subsequent analysis of the Reversibility by Treatments interaction showed treatments to be significant $(p<.01)$ for both reversibles and nonreversibles (Figure 1B).

Practice with a small number of mismatch types clearly led to marked changes in subjects' processing strategies from early to late trials (Figure 1A vs. 1B). In nonreversibles, the only difference was in the order of comparison, which changed from LS-V-LO to LS-LO-V

Table 2

Mean RT, Standard Error of the Mean (in Milliseconds), and Percentage Errors for Each Treatment Type on (A) Early (Blocks 1-2) and (B) Late (Blocks 3-5) Trials

\begin{tabular}{|c|c|c|c|c|c|c|c|c|}
\hline & & & \multicolumn{3}{|c|}{ (A) Early } & \multicolumn{3}{|c|}{ (B) Late } \\
\hline & & & $\begin{array}{c}\text { Mean } \\
\text { RT }\end{array}$ & $\begin{array}{c}\text { SE } \\
\text { Mean }\end{array}$ & $\begin{array}{c}\text { Percent } \\
\text { Error }\end{array}$ & $\begin{array}{c}\text { Mean } \\
\text { RT }\end{array}$ & $\begin{array}{c}\text { SE } \\
\text { Mean }\end{array}$ & $\begin{array}{c}\text { Percent } \\
\text { Error }\end{array}$ \\
\hline \multirow[b]{4}{*}{ Reversible } & True & & 1412 & 105 & 2.3 & 1384 & 87 & 3.9 \\
\hline & False & $\mathrm{s}$ & 1582 & 159 & 14.2 & 1277 & 66 & 7.1 \\
\hline & & $v$ & 1583 & 115 & 3.5 & 1199 & 70 & 2.3 \\
\hline & & o & 1559 & 141 & 0 & 1229 & 80 & 7.1 \\
\hline \multirow[t]{4}{*}{ Passive } & True & & 1425 & 92 & 8.3 & 1451 & 86 & 3.9 \\
\hline & False & $s$ & 1647 & 178 & 10.7 & 1306 & 81 & 14.2 \\
\hline & & $\mathbf{v}$ & 1596 & 121 & 3.5 & 1168 & 63 & 4.7 \\
\hline & & o & 1683 & 116 & 3.5 & 1293 & 79 & 2.3 \\
\hline \multirow{8}{*}{$\begin{array}{l}\text { Nonreversible } \\
\text { Passive }\end{array}$} & True & & 1264 & 78 & 4.7 & 1190 & 87 & .7 \\
\hline & False & $\mathrm{s}$ & 1184 & 78 & 0 & 1159 & 62 & 0 \\
\hline & & $\mathbf{v}$ & 1233 & 66 & 3.5 & 1287 & 74 & 0 \\
\hline & & 0 & 1408 & 84 & 7.1 & 1200 & 74 & 9.5 \\
\hline & True & & 1267 & 80 & 3.5 & 1142 & 79 & .7 \\
\hline & False & s & 1156 & 113 & 7.1 & 1069 & 83 & 7.1 \\
\hline & & v & 1275 & 73 & 7.1 & 1272 & 84 & 2.3 \\
\hline & & o & 1386 & 86 & 0 & 1168 & 85 & 4.7 \\
\hline
\end{tabular}




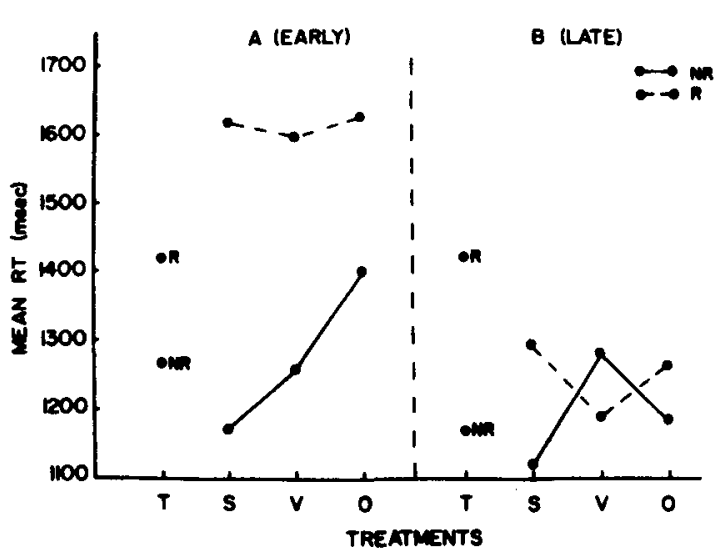

Figure 1. Response latencies to true sentences and three mismatch types for reversible and nonreversible stimuli on early (1A) and late (1B) trials.

(in Figure 1B: $s<0<\mathrm{v}, \mathrm{s}<\mathrm{T}=\mathrm{o}<\mathrm{v}, \mathrm{p}<.05$ ). In reversibles, however, the major change was in the relationship between true and false latencies: on early trials true latencies were significantly faster than those to all mismatch types, while on later trials this relationship was reversed, suggesting that with practice subjects abandoned the exhaustive strategy they began with. The pattern of latencies to the three mismatch types in Figure 1B is identical to that obtained in Experiment I for $\mathrm{s}, \mathrm{v}$, and o-mismatches in reversibles, and again is compatible with several possible strategies. Again, however, it does not confirm Glucksberg et al.'s finding of a V-GS-GO order of comparison, despite the reduction in number of mismatch types. Finally, with regard to the effect of sentence voice on processing, the data in Table 2 support the conclusions suggested by the results of Experiment I: the syntactic factor of voice was bypassed in the processing of both true and false nonreversibles, and false reversibles, but not true reversibles. Voice was therefore not a factor in processing unless (a) the sentence and picture constituents all matched, and (b) the subjects and objects were reversible.

\section{GENERAL DISCUSSION}

The results of Experiments I and II indicate that subjects' specific encoding and comparison strategies in verification tasks can vary not only across, but also within, task contexts. Contrary to the findings of Glucksberg et al., subjects do not necessarily adopt a verb-first comparison strategy when processing a visual input. In nonreversibles the order of comparison was LS.V.LO, or, after practice with a small number of mismatch types, an order in which the verb was processed last (LS-LO-V). In reversibles, the verb was processed most quickly, but because RTs to s- and o-mismatches were equal rather than ordered one can not unambiguously conclude that the verb was in fact processed first; it is also possible that reversible pictures were processed in an LS.V.LO order, but that comparison did not terminate with a subject-mismatch because of the confusability of the subject and object. Such a strategy is certainly compatible with the finding in Experiment II that on early trials true responses were significantly faster than false responses, suggesting that subjects began with an exhaustive comparison strategy.

The general comprehension model proposed by Glucksberg et al. can easily accommodate task-specific differences in encoding and comparison strategies, but the finding that reversibility has a significant effect on false latencies raises a serious question about the generality of their model. Glucksberg et al. assume reversibility information is not utilized unless the three constituents match, but in both Experiments I and II reversibility clearly influenced latencies to false as well as true sentences. Finally, the results of the present studies strongly suggest that sentences were encoded as heard in their surface structure format, but that the succeeding pictures were not necessarily encoded in an identical format prior to comparing the two inputs. Glucksberg et al. concluded that in their studies subjects encoded both inputs in a verb-first list format, regardless of voice or reversibility. The results of the present studies indicate this is not inevitable when sentences are presented auditorily, regardless of the number of mismatch types. It remains to be determined whether allowing subjects unlimited time to encode the first stimulus input, and/or reducing the size of the stimulus sets from which lexical constituents are drawn, would be sufficient conditions for eliciting an unambiguous verb-first processing strategy, even when stimulus constituents are nonreversible.

\section{REFERENCES}

Bever, T. G. The cognitive bases for linguistic structures. In J. R Hay es (Ed.), Cognition and the development of language. New York: John Wiley and Sons, Inc., 1970.

Clark, H. H., \& Chase, W. G. On the process of comparing sentences against pictures. Cognitive Psychology, 1972, 3, 472-517.

Glucksberg, S., Trabasso, T., \& Wald, J. Linguistic structures and mental operations. Cognitive Psy chology, 1973, 5, 338-370.

Huttenlocher, J., \& Higgins, E. T. On reasoning, congruence, and other matters. Psy chological Review, 1972, 79, 420-427.

Olson, D. R., \& Filby, N. On the comprehension of active and passive sentences. Cognitive Psy chology, 1972, 3, 361-381.

Trabasso, T. Mental operations in language comprehension. In J. B. Carroll and R. O. Freedle (Eds.), Language comprehension and the acquisition of knowledge. Washington: Winston. 1972.

Wannemacher, J. T. Processing strategies in picture-sentence verification tasks. Memory \& Cognition, 1974, 2, 554-560.

(Received for publication January 29, 1975 Revision received March 21, 1975.) 